\title{
ELASTIC-PLASTIC ANALYSIS OF CRACKS IN PRESSURE-SENSITIVE MATERIALS
}

\author{
P. Dongt and J. PAN \\ Department of Mechanical Engineering and Applied Mechanics. The University of \\ Michigan. 2250 G. G. Brown, Ann Arbor, MI 48109. U.S.A.
}

\author{
(Receted 29 May 1990; in retised form 10 February 1991)
}

\begin{abstract}
In this paper we present an elastic-plastic analysis of the small-scale yielding crack-tip ficlds for pressure-sensitive materials. Mode I loading and plane-strain conditions are assumed. The yield criterion is chosen to be a linear combination of the effective stress and the hydrostatic stress. For power-law hardening materials, our elastic-plastic finite element analysis shows that HRRtype crack-tipe fields are obtained not only for $\mu \leqslant \mu_{\mathrm{him}}$, but also for a range of $\mu>\mu_{\mathrm{hm}}$. Here $\mu_{\mathrm{km}}$ is referred to as the limit value of a pressure sensitivity parameter $\mu$ in $\mathrm{Li}$ and Pan $[J$. Appl. Alech. 57. 40-49 $(1990)]$. When elastic-perfectly plastic behavior is considered. the finite element results show that clastic sectors exist near the crack tip. The sizes of the elastic sectors vary with $\mu$. Plastic zones are also given for different values of the pressure sensitivity parameter $\mu$ and the hardening exponent $n$. The parameter $\mu$ has a significant effect on the plastic zone sizes and shapes. The contribution of the hydrostatic stress in the yield criterion causes the plastic zone boundary in front of the crack tip to extend much farther than that in an incompressible material.
\end{abstract}

\section{INTRODUCTION}

Although the general theoretical framework of the constitutive laws for pressure-sensitive materials such as rocks, concrete, soils, etc., are well laid out in the literature (for example, see Drucker and Pralger, 1952; Wu, 1966; Rudnicki and Rice, 1975; Spitzig and Richmond, 1979; and Needleman and Rice. 1978), the consequences of pressure-sensitive yielding and plastic dilatation, as far as crack-tip mechanics are concerned, have remained relatively unexplored. This becomes increasingly necessary due to the fact that some new materials, for example, toughened structural polymers and ceramics, show great potential in modern structural applications. In this class of materials, appreciable volumetric plastic deformations hatve been reported in the past. It is believed that for this type of materials, the pressure-sensitive yielding arises in part from basic flow mechanism in polymers, microcracking and phase transformation in some ceramics, and in part from cavitation and craze formation in polymers.

So motivated, $\mathrm{Li}$ and Pan (1990:a) recently investigated the asymptotic crack-tip stress and strain fields for pressure-sensitive dilatant materials. They assumed a simple hydrostatic stress-dependent yielding criterion (Drucker and Prager, 1952). The corresponding cracktip stress and strain fields have been obtained for a limited range of pressure sensitivity parameter $\mu$ under plane-strain conditions. But many questions remain to be answered. For instance, $\mathrm{Li}$ and Pan (1990a) identified a limit value, $\mu_{\mathrm{limn}}$, of the pressure sensitivity parameter $\mu$ for each hardening exponent, $n$, such that for $\mu \geqslant \mu_{\text {lim }}$, the numerical method for obtaining the HRR-type crack-tip fields starts diverging. For the perfectly plastic case $(n \rightarrow \infty), \mu_{\mathrm{lum}}$ is $\sqrt{3} / 2$. The existence of $\mu_{\text {lim }}$ might be due to the assumption of the constitutive law under plane-strain constraint conditions. To address the above and many other issues regarding the stress and strain fields near a crack tip, it is necessary to carry out a full-field clastic-plastic analysis. In this report, we concentrate on the mode I crack-tip fields under plane-strain conditions. The results on the corresponding plane-stress crack-tip fields are reported in Li and Pan (1990b) and Ben Aoun and Pan (1991).

\section{MATERIAL IDEALIZATION}

Throughout this investigation, we adopt a simple pressure-sensitive yield criterion that contains two stress invariants, the effective stress $\sigma_{e}$ and the hydrostatic stress $\sigma_{m}$, in such

+ Current address: Edison Welding Institute, 1100 Kinnear Road. Columbus, OH 43212. U.S.A. 
a way that

$$
\dot{\psi}\left(\sigma_{1}\right)=\sigma_{e}+\sqrt{3} \mu \sigma_{m}=Q
$$

where

$$
\sigma_{\mathrm{e}}=\left(3 s_{i j} s_{i,} 2\right)^{12}, \quad s_{i j}=\sigma_{i j}-\sigma_{m} \dot{\delta}_{i j}, \quad \sigma_{m}=\sigma_{k k} / 3
$$

and $\psi\left(\sigma_{i j}\right)$ represents the current yield surface in the stress space. The material constant $\mu$ measures the pressure sensitivity of yielding. The characteristic yield strength $Q$ can be taken to depend upon the plastic work $W^{p}$. Further information on the pressure-sensitive criterion can be found in Drucker and Prager (1952); its applications in metals, polymers and ceramics are discussed in Drucker (1973), Spitzig and Richmond (1979), and ReyesMorel and Chen (1988). follows :

Using Li and Pan's notation, we can introduce the generalized effective stress $\sigma_{4 e}$ as

$$
\sigma_{y e}=\sigma_{e}+\sqrt{3} \mu \sigma_{m}
$$

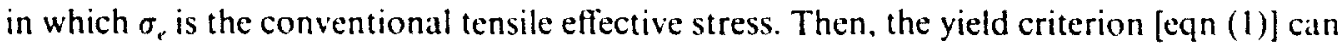
be restated as

$$
\sigma_{v e}=\sigma_{c}+\sqrt{3} \mu \sigma_{m}=Q\left(W^{\prime}\right)
$$

The outward normal tensor $S_{1 /}$ of the yicid surface in the stress space is

$$
S_{i \prime}=\frac{i \psi\left(\sigma_{i \prime}\right)}{i \sigma_{i j}}=\frac{i \sigma_{u r}}{\partial \sigma_{1 j}}=\frac{3 s_{11}}{2 \sigma_{\nu}}+\frac{\mu}{j / 3} \delta_{11}
$$

The pressure-sensitivity parameter, $\mu$, of the initial yield surface can be obtained from the compressive yield strength $\sigma_{\imath}$ and the tensile yield strength $\sigma_{\imath}$ (Necdleman and Rice, 1978):

$$
\mu=\sqrt{3} \frac{\sigma_{c}-\sigma_{t}}{\sigma_{c}+\sigma_{t}}
$$

As the strength differential between compression and tension increases, an extreme case is reached at $\sigma_{c}=\infty$ in compression, and $\mu$ becomes $\sqrt{3}$. When $\mu$ is larger than $\sqrt{3}$, determination of $\mu$ based on eqn (6) is no longer appropriate under the current assumed yielding criterion of eqn (1). A method for determining $\mu$ for toughened ceramics is devised by Chen and Reyes-Morel (1986).

In this study, the plastic behaviour of the pressure-sensitive materials is assumed to obey the fictitious power-law stress-strain relation when $\sigma_{g e}$ is larger than $\sigma_{0}$ :

$$
\frac{\varepsilon_{g e}^{p}}{\varepsilon_{0}}=\alpha\left(\frac{\sigma_{g e}}{\sigma_{0}}\right)^{n}-\alpha \frac{\sigma_{g e}}{\sigma_{0}}
$$

where $\varepsilon_{y e}^{p}$ represents the generalized effective plastic strain. Since we adopt the normality of plastic flow, the generalized effective plastic strain rate equals the tensile effective plastic strain rate. The tensile effective plastic strain, which provides a measure of the plastic distortion, is obtained from integration over the deformation history based on the tensile effective plastic strain rate defined as $\dot{\varepsilon}_{e}^{p}=\left((2 / 3) \dot{e}_{i j}^{p_{i}} \dot{e}_{i}^{p}\right)^{1,2}$. Here $\dot{e}_{i j}^{p}$ represents the deviatoric plastic strain rate. The corresponding deformation plasticity version of the constitutive law can be found in $\mathrm{Li}$ and $\mathrm{Pan}$ (1990a). For the elastic-perfectly plastic case, $\sigma_{g e}$ takes the constant value of $\sigma_{0}$, the yield stress in uniaxial tension. In eqn (7), $\varepsilon_{0}$ is the yield strain in uniaxial tension. Within the context of the small-strain flow theory of plasticity, the total strain rate tensor in the plastic range can be decomposed into an elastic and a plastic part: 


$$
\dot{\varepsilon}_{i j}=\dot{\varepsilon}_{i j}^{i}+\dot{\varepsilon}_{i j}^{p} \text {. }
$$

The elastic strain rate tensor $\dot{\varepsilon}_{i j}^{e}$ is related to the stress rate tensor $\dot{\sigma}_{i j}$ through a constant, isotropic, positive-definite elasticity tensor $C_{i, k l}$ as

$$
\dot{\sigma}_{i j}=C_{i j k l} \dot{\varepsilon}_{k l}^{e}
$$

The plastic strain rate tensor $\dot{\varepsilon}_{i j}^{p}$ is normal to the yield surface and the flow rule takes the form

$$
\dot{\varepsilon}_{i j}^{p}=\lambda\left(\frac{3}{2} \frac{s_{i j}}{\sigma_{e}}+\frac{\mu}{\sqrt{3}} \delta_{i j}\right)
$$

where $i>0$ under plastic loading conditions.

By using eqns (1)-(10) the constitutive law for a material currently experiencing elasticplastic deformation can be obtained as

$$
\dot{\sigma}_{i j}=C_{i j k l}^{*} \dot{\varepsilon}_{k l}=\left[C_{i j k l}-\frac{C_{t j p q} S_{p q} S_{m n} C_{m n k l}}{S_{r l} C_{r t u v} S_{w i}+{ }_{9}^{4} \sigma_{g e}^{2} H^{\prime}}\right] \dot{\varepsilon}_{k l}
$$

In the above, $H^{\prime}=\mathrm{d} \sigma_{g e} / \mathrm{d} \varepsilon_{g e}^{p}$ can be obtained from eqn (7) for the power-law hardening materials and $H^{\prime}=0$ for elastic-perfectly plastic materials. The plane-strain constraints are imposed through the requirement that $\dot{\varepsilon}_{3 i} \equiv 0$. For simplicity, $\mu$ is assumed to be constant in the entire deformation history in this investigation.

\section{FINITE ELEMENT ANALYSIS}

\subsection{Computational model}

We consider a crack in an elastic-plastic solid with the reference coordinate systems depicted in Fig. 1; the Cartesian coordinates $x_{1}$ and $x_{2}$ and the polar coordinates $r$ and $\theta$ are centered at the tip. We consider the so-called small-scale yielding problem where the plastic zone near the tip is small compared to the relevant length parameters such as the crack length under remote loading. The small-scale yielding problem was modeled by considering the crack in the circular domain of radius $r_{10}$. To take advantage of the mode I symmetry of the problem, only the upper half of the circular domain was considered and discretized using eight-node serendipity elements. In the immediate crack-tip region, we used a ring of 20 wedge-shaped elements of size $r_{i}$. The crack-tip elements with collapsed nodes were equally distributed from 0 to $\pi$ and surrounded by 24 semi-circular strips of elements generated by a logarithmic scale in the $r$ direction. The entire model consists of a total of 500 isoparametric elements, and $r_{i} / r_{0} \approx 10^{-5}$ is used in the calculations.

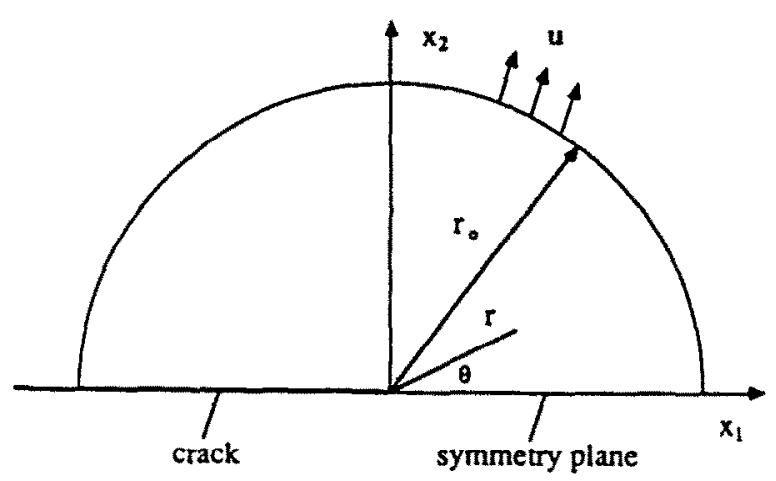

Fig. I. Problem definition and the coordinate conventions. 
The displacement due to the leading singular term of the linear elastic asymptotic solution of the crack-tip field.

$$
u_{1}=\frac{k_{1}}{2 G} \sqrt{2 \pi} i_{i}(\theta, \cdots) . \quad i=1.2
$$

is specitied as the boundary condition at the outermost boundary $r=r_{11}$ of the domain. Here, $G$ represents the shear modulus, $v$ represents the Poisson ratio, $K$, denotes the mode I stress intensity factor of the far-field. and $i_{i}(\theta, v)$ are the dimensionless displacement functions associated with the elastic singularity and depend only on the orientation for a given elastic material. The loading is applied through the stress intensity factor. $K_{1}$. which can be viewed as an amplitude factor in eqn (12). The remote load intensity can also be expressed in terms of the well-known $J$ integral (Rice, 1968) as

$$
J=\frac{1-v^{2}}{E} K
$$

where $E$ is Young modulus, to facilitate later discussions.

\subsection{Numerical procedurc}

Within the context of the small-strain approach, an incremental plasticity theory was employed with the pressure-sensitive yield criterion and the associated flow rule. $A$ displacement-based finite element method with an iterative procedure based on a modified Newton Raphson method was used in the analysis. The finite element equations were derived from the principle of virtual work. At time $t+\Delta t$, this takes the form

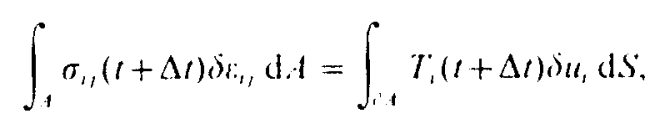

where $\sigma_{i}(t+\Delta t)$ represents the Cauchy stress tensor, which satisfies the equilibrium conditions at time $t+\Delta t$, and $T_{i}(t+\Delta t)$ is the imposed traction vector on the boundary $i d$ of domain $A$. In adddition, $\delta u$, represents the virtual displacement field that vatnishes on the part of the boundary where the displacement is specified, and $\delta \varepsilon_{i}$, is the associated smallstrain tensor. Here, time $t$ is used as a convenient variable to represent different loading levels. Linearizing eqn (14) with respect to the equilibrium conliguration at lime $t$ and introducing the finite element approximation, we obtain the following incremental eyuilibrium equations in matrix notation:

$$
\mathbf{K}_{r} \Delta \mathbf{U}=\mathbf{F}(t+\Delta t)-\mathbf{P}(t)
$$

where $\Delta \mathbf{U}=\mathbf{U}(t+\Delta t)-\mathbf{U}(t)$ is the vector of incremental displacements at the nodal points, $\mathbf{K}_{I}=\int_{A} \mathbf{B}^{\prime} \mathbf{D B} \mathrm{d} A$ is the tangent stiffness matrix corresponding to the configuration at time $t$ (B is the strain displacement matrix and $\mathbf{D}$ the material constitutive matrix of the elastic plastic material), $\mathbf{F}(t+\Delta t)$ is the vector of the applied external loads at time $t+\Delta t$, and $\mathbf{P}(t)=\int_{A} \mathbf{B} \sigma(t) \mathrm{d} A$ is the equivalent foree vector of the element stresses at time $t$.

The loading is applied through the mode I stress intensity factor, $K$, which enters the far-ficld displacement boundary conditions [eqn (12)] as an amplification factor. An iterative Newton-Raphson procedure is employed to solve the incremental equilibrium equations [eqn (15)] for each load increment, such that for the $k$ th equilibrium iteration at time $t+\Delta t$. the Euclidean norm

$$
\Delta \mathbf{R}^{k}=\mathbf{F}(t+\Delta t)-\mathbf{P}^{k-1}(t+\Delta t)
$$




$$
\frac{\left\|\Delta \mathbf{R}^{k}\right\|}{\| \mathbf{F}_{i}} \leqslant T O L
$$

where $T O L$ is a small preset tolerance. It is important that the stress evaluation, which can be written as

$$
\sigma_{i j}^{k}(t+\Delta t)=\sigma_{i j}(t)+\int_{\varepsilon_{i,}(t)}^{c_{t,}^{k}(t+\Delta t)} D_{i j k l} \mathrm{~d} \varepsilon_{k i}
$$

is performed by integrating from the values of the last accepted equilibrium state to the current state of iteration $k$, so that the final results are not affected by errors introduced during intermediate iterations (Bathe and Cimento, 1980). The incremental processes are continued until a steady stress state at the crack tip is observed. At all times the maximum extent of the plastic zone around the crack tip is smaller than $1 / 100$ of $r_{0}$ to preserve smallscale yielding conditions (Dong and Pan, 1990a,b). For the results reported here, $v$ is taken as 0.3 , and the ratio $E / \sigma_{0}$ as 500 .

\section{ASYMPTOTIC CRACK-TIP FIELDS}

\subsection{Poncer-lan hardening solutions}

For power-law hardening Mises materials, the asymptotic crack-tip stress and strain fields possess the well-known HRR singularity (Hutchinson, 1968a,b; Rice, 1968 ; and Rice and Rosengren, 1968). By the same token, Li and Pan (1990)i,b) show that the asymptotic crack-tip stress, strain, and displatement fields in power-law hardening pressure-sensitive materials can be written as:

$$
\begin{aligned}
& \sigma_{i j}=\sigma_{0}\left[\begin{array}{c}
J \\
\alpha \sigma_{0} \varepsilon_{0} I(n, \mu) r
\end{array}\right]^{1 /(n+1)} \tilde{\sigma}_{i j}(0 ; n, \mu) \\
& \varepsilon_{i j}=\alpha \varepsilon_{00}\left[\frac{J}{\alpha \sigma_{0} \varepsilon_{0} I(n, \mu) r}\right]^{n /(n+1)} \tilde{\varepsilon}_{i j}(\theta ; n, \mu) \\
& u_{i}=\alpha \varepsilon_{0,0} r\left[\frac{J}{\alpha \sigma_{0} \varepsilon_{0} I(n, \mu) r}\right]^{n /(n+1)} \tilde{u}_{i}(\theta ; n, \mu) .
\end{aligned}
$$

In the above, the dimensionless function $l$ and the dimensionless angular functions $\tilde{\sigma}_{i j}, \tilde{\varepsilon}_{i j}$, and $i_{i}$ depend on the stratin hardening exponent, $n$, the pressure sensitivity factor, $\mu$, and the conditions of plane-strain or plane-stress. These angular functions are normalized by setting the maximum value of the dimensionless generalized effective stress $\tilde{\sigma}_{g e}$ equal to unity. The asymptotic solutions were obtained by $\mathrm{Li}$ and $\mathrm{Pan}$ (1990a) for a limited range of $\mu$ for each $n$ under plane-strain conditions. In order to investigate the reason for the existence of $\mu_{\mathrm{lm}}$, we have carried out full-field finite element computations. The numerical results are presented in the following.

For the case of $n=3$, the crack-tip stress fields at $r / r_{p} \approx 10^{-3}$ as functions of 0 for $\mu=0,0.1$ and 0.2 are shown in Fig. 2. Here $r_{p}$ represents the extent of the plastic zone from the crack tip at $\theta=0$. At $r / r_{p} \approx 10^{-3}$, steady state asymptotic solutions were obtained in our computations for hardening materials. All the stress components are normalized by $\sigma_{0}\left[J /\left(x \sigma_{0} \varepsilon_{0} r\right)\right]^{1 /(1+n)}$. For $\mu=0$, the stress field shown in Fig. $2 \mathrm{a}$ is in agreement with the corresponding HRR field of Hutchinson (1968a,b) and Rice and Rosengren (1968). As shown in Fig. 2a and b, where $\mu$ increases from 0 to 0.1 , the difference of $\sigma_{\theta \theta}$ and $\sigma_{r r}$ ahead of the crack tip decreases and nearly vanishes. This means that the stress state ahead of the crack tip approaches pure hydrostatic tension as $\mu$ increases. This trend is the same as that of the asymptotic analysis of $\mathrm{Li}$ and Pan (1990a). 

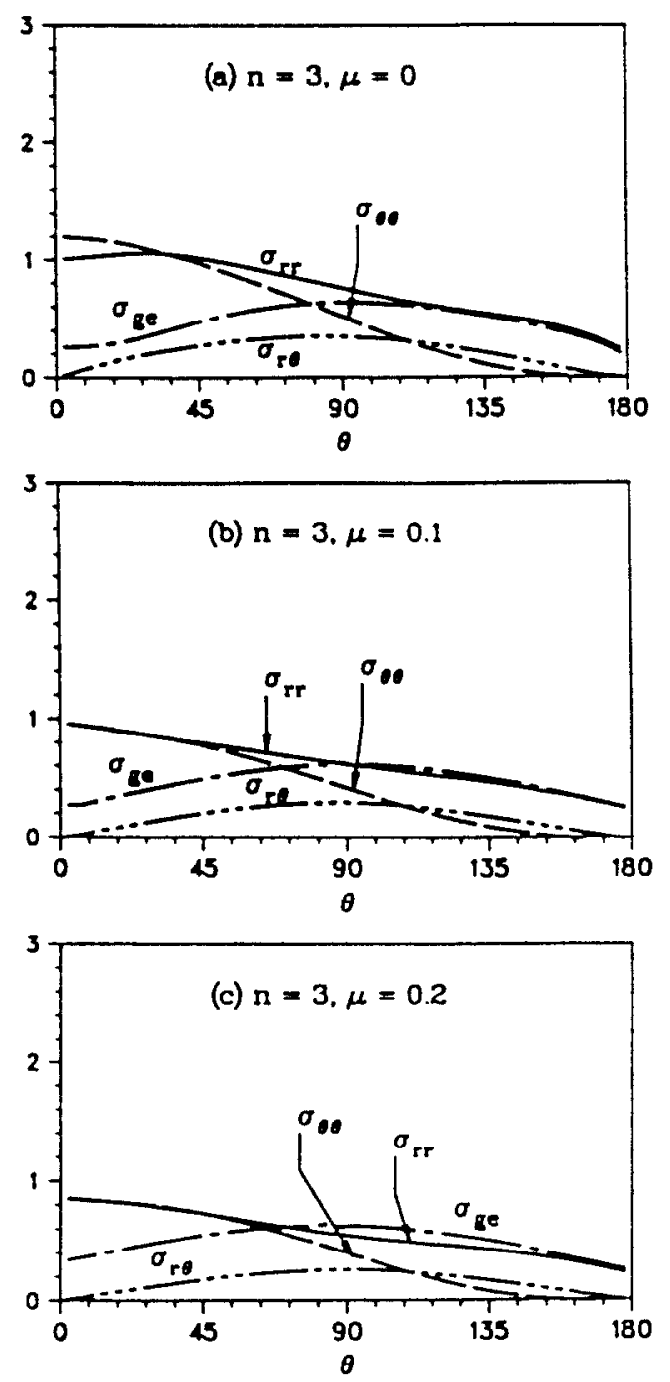

I.ig. 2. Crack-tip stress liclds for $n=3:$ (a) $\mu=0$, (b) $\mu=0.1$, (c) $\mu=0.2$.

It should be noted that the limit value $\mu_{\mathrm{hm}}$ for $n=3$ is supposed to be $0 .(1)$ according to the asymptotic analysis of $\mathrm{Li}$ and Pan (1990a). Figure $2 \mathrm{c}$ shows the results for $\mu=0.2$. which are more than twice as much as $\mu_{\text {lum }}$ for $n=3$. The stress state ahead of the crack tip for $\mu=0.2$ seems to stay in nearly pure hydrostatic tension. However, the magnitudes of the normalized stresses, $\sigma_{i m}$ and $\sigma_{r r}$ ahead of the tip are less than those of $\mu=0.1$. More significantly, the stress field for $\mu=0.2$ is still of HRR-type based on the numerical results of the radial dependence of the stresses, whereas $\mathrm{Li}$ and Pan (1990a) were unable to find any solutions for $\mu \geqslant \mu_{\text {lim }}$.

The same trend is also observed for the case of $n=10$. Figure 4 shows the angular functions of the normalized stresses for $\mu=0,0.2$ and 0.4 . The angular functions of the normalized stresses in Fig. $4 \mathrm{a}$ and $\mathrm{b}$ for $\mu=0$ and 0.2 agree well with the corresponding asymptotic solutions of Hutchinson (1968a,b), Rice and Rosengren (1968), and Li and Pan (1990a). Note that $\mu_{\text {lim }}$ for $n=10$ is 0.344 . Here we still can find the HRR-type crack-tip field as shown in Fig. $4 \mathrm{c}$ for $\mu=0.4$.

The corresponding plastic zones normalized by $\left(1-v^{2}\right) \sigma_{0}^{2} / J E$ are plotted in Figs 3 and 5 for $n=3$ and $n=10$, respectively. These figures show that for both $n=3$ and $n=10$ the normalized plastic zones expand and shift to the front of the tip as $\mu$ increases. The trend of the plastic zone shape change with the increase of $\mu$ differs from that of the generalized effective stress contours of the asymptotic analysis of Li and Pan (1990a). 

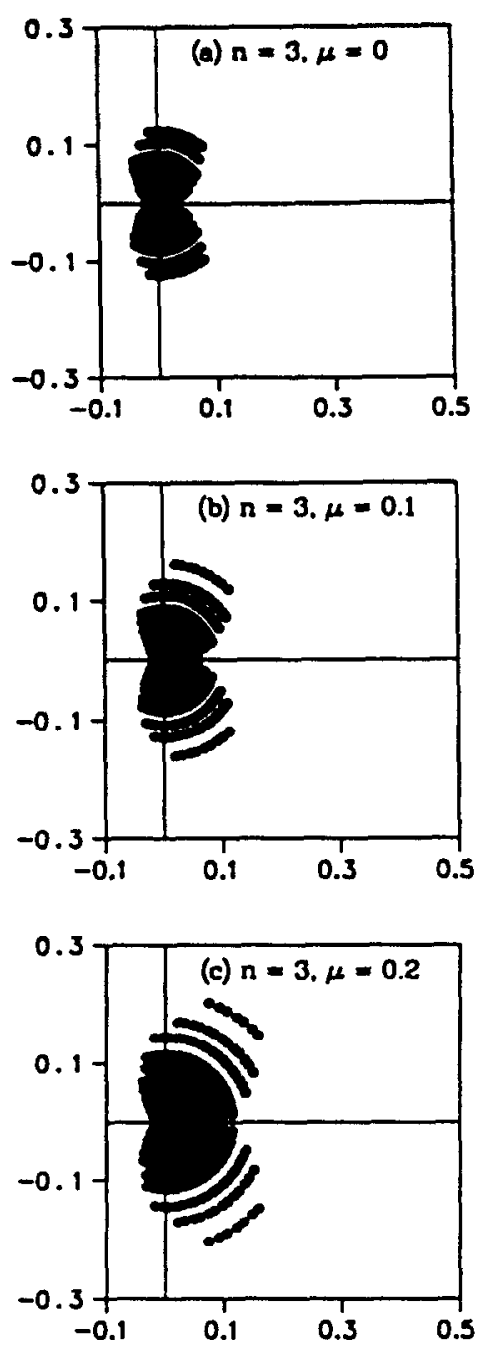

Fig. 3. Plastic zones normalized by $\left(1-v^{2}\right) \sigma_{i}^{2} / J E$ for $n=3$ : (a) $\mu=0$. (b) $\mu=0.1$. (c) $\mu=0.2$.

\subsection{Perfectly plastic solutions}

As the hardening exponent $n$ approaches $\infty$, the perfectly plastic limit is realized. It seems that simple extrapolations from the low hardening limit of the power-law solutions may not be entirely appropriate under certain circumstances. Some relevant discussions can be found in Gao (1980). Nemat-Nasser and Obata (1984) and Dong and Pan (1990a,b) for Mises materials. Nevertheless, some fundamental effects of the pressure-sensitive yielding on the crack-tip fields can still be brought to light with the assumption that the material surrounding the crack tip is fully yielded at all angles. In doing so, $\mathrm{Li}$ and $\mathrm{Pan}$ (1990a) constructed a slip-line field for $\mu<\mu_{\text {lim }}$. The resulting slip-line grid is formed by two nonorthogonal families of characteristic lines. However, they failed to identify the solution for $\mu=\mu_{\text {lim }}$. In the following, we will present a slip-line field for the limit case in order to shed some light on the physical interpretations of $\mu_{\lim }$ for perfectly plastic materials.

4.2.1. Rigid perfectly plastic solution for $\mu=\mu_{\text {fim }}$. The asymptotic form of the equilibrium equations with respect to the polar coordinates (Fig. 1) can be written as:

$$
\begin{gathered}
\sigma_{r r}-\sigma_{\theta \theta}+\frac{\mathrm{d} \sigma_{r \theta}}{\mathrm{d} \theta}=0 \\
2 \sigma_{r \theta}+\frac{\mathrm{d} \sigma_{\theta \theta}}{\mathrm{d} \theta}=0
\end{gathered}
$$



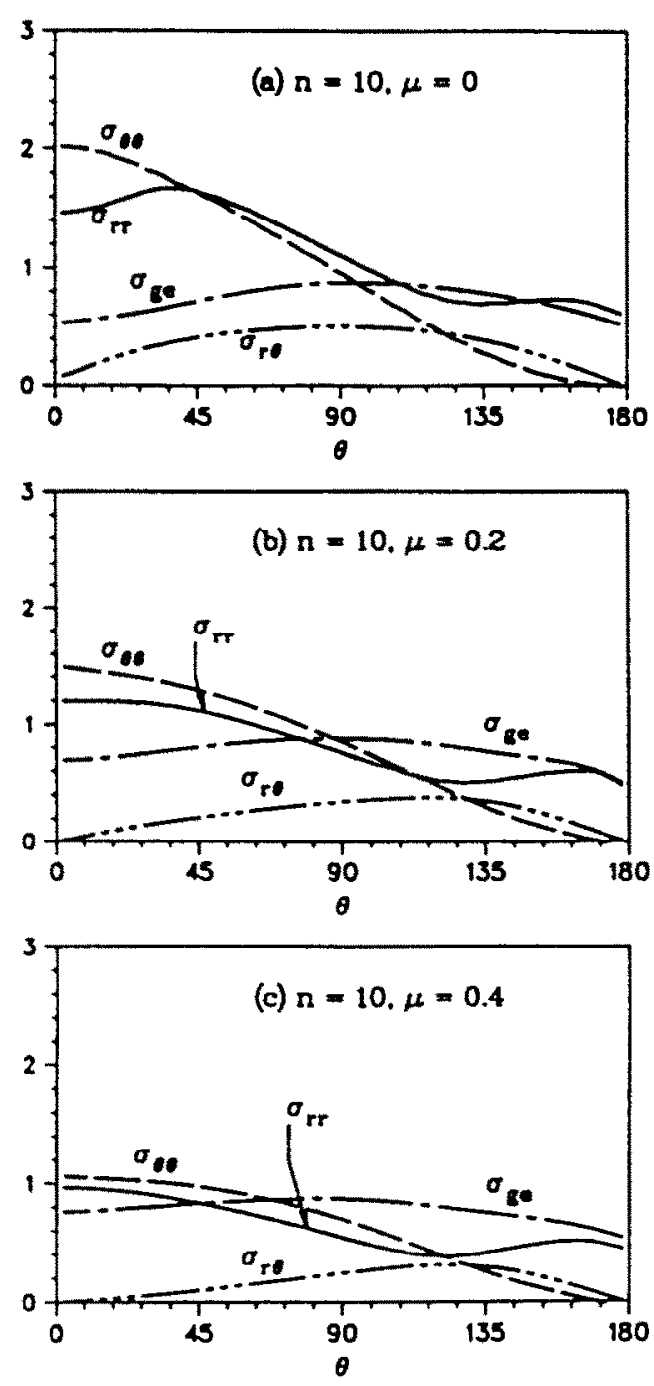

Fig. 4. Crack-tip stress ficids for $n=10$ : (a) $\mu=0$, (b) $\mu=0.2$, (c) $\mu=0.4$.

The pressure-sensitive yicld criterion can be rewritten as:

$$
\left[\left(\frac{\sigma_{r r}-\sigma_{\theta \theta}}{2}\right)^{2}+\sigma_{r \theta}^{2}\right]^{1,2}+\sin \phi \frac{\sigma_{r r}+\sigma_{m t}}{2}=\frac{\sigma_{0}}{\sqrt{3-\mu^{2}}},
$$

where

$$
\sin \phi=\frac{\mu}{\left[1-\frac{1}{3} \mu^{2}\right]^{12}}
$$

It is convenient to rewrite the yield criterion, eqn (24), in terms of the normal stress $\sigma$ and the shear stress $\tau$ in the Mohr plane (Fig. 6b):

$$
\tau=c-\sigma \tan \phi .
$$

In eqn (26), we have introduced another parameter $c$ : 

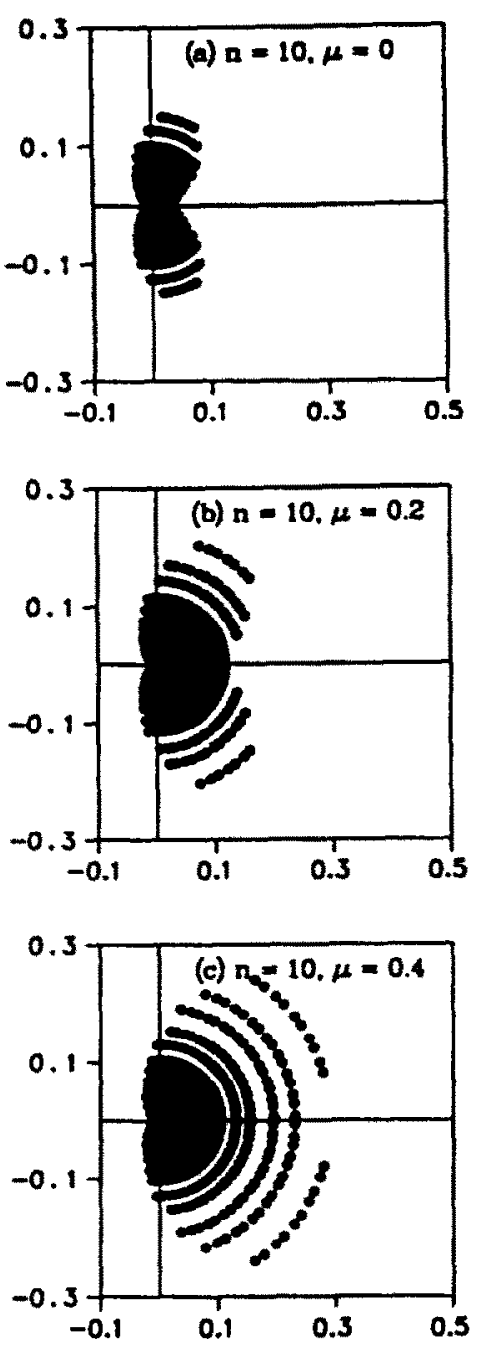

Fig. 5. Plastic zones normalized by $\left(I-v^{2}\right) \sigma_{0}^{2} / J E$ for $n=10$; (a) $\mu=0$. (b) $\mu=0.2$, (c) $\mu=0.4$.

$$
c=\frac{\sigma_{0}}{\sqrt{3}\left[1-\frac{4}{3} \mu^{2}\right]^{1 / 2}}
$$

Evidently, eqn (26) is in the same form of the well-known Coulomb yield criterion, and $c$ and $\phi$ are referred to as the cohesion and the angle of internal friction, respectively.

By means of combining eqns (22)-(24), it becomes apparent that there exist two types of plastic crack-tip sectors [see Rice (1982) for the general framework of asymptotic cracktip fields for perfectly plastic materials]. One is the constant stress sector, and the other is the curved fan sector. Within the constant stress sector, the characteristic grid is generated by two non-orthogonal families of parallel lines, namely $\alpha$ lines and $\beta$ lines. Within the curved fan sector, the grid is generated by one family of radial lines and another family of spiral curves. In both cases, the two families of characteristics intersect each other at $\pi / 2+\phi$, as shown in Fig. 7. On the plane along the characteristic lines, the normal stress $\sigma$ and the shear stress $\tau$ satisfy eqn (26).

If $\mu=0$, the yield surface is described by two horizontal lines $(\phi=0)$ intersecting the $\tau$-axis at $\tau= \pm \sigma_{0} \sqrt{3}$ (Fig. 6a). and incompressible yielding results. The two families of the characteristics are $\pi / 2$ apart (Figs 7 and 8 a), and become the slip lines of the conventional slip-line theory for incompressible materials. If $\mu=\sqrt{3} / 2$, the two inclined lines describing the yiedd surface become one single vertical line $(\phi=\pi / 2)$ intersecting the $\sigma$-axis at $2 \sigma / 3$ (Fig. $6 \mathrm{c}$ ), and pure normal stress-dependent yielding results. The two families of the stress 


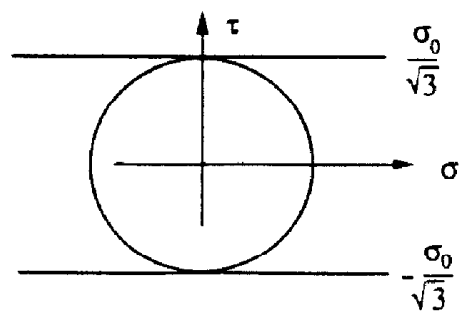

(a) $\mu=0$

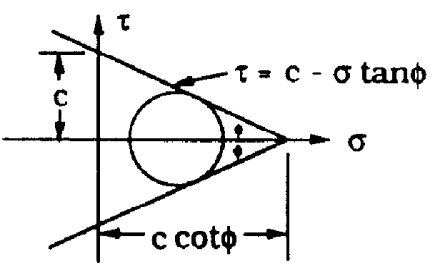

(b) $0<\mu<\sqrt{3} / 2$

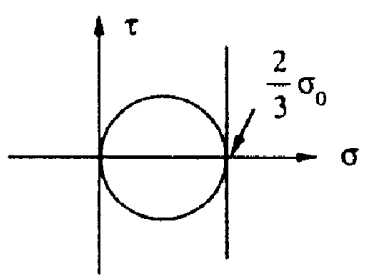

(c) $\mu=\sqrt{3} / 2$

Fig. 6. Yield surfaces displayed in the Mohr plane : (a) $\mu=0$, (b) $0<\mu<\sqrt{3 / 2}$, (c) $\mu=\sqrt{3} / 2$.

characteristics converge to one. The same conclusion can also be reached by examining the governing equations using the well-known determinant method. As $\mu$ changes from 0 to $\sqrt{3} / 2$, the nature of the governing equations changes from hyperbolic to parabolic everywhere around the crack tip at $\mu=\sqrt{3 / 2}$. The assembly of the characteristics for the cracktip stress field is shown in Fig. 8c, and the stresses are of the following form:

for $0 \leqslant 0<\pi 2$ :

$$
\begin{gathered}
\frac{\sigma_{r r}}{\sigma_{0}}=\frac{\sigma_{\theta+t}}{\sigma_{0}}=之, \\
\frac{\sigma_{r \theta}}{\sigma_{0}}=0,
\end{gathered}
$$

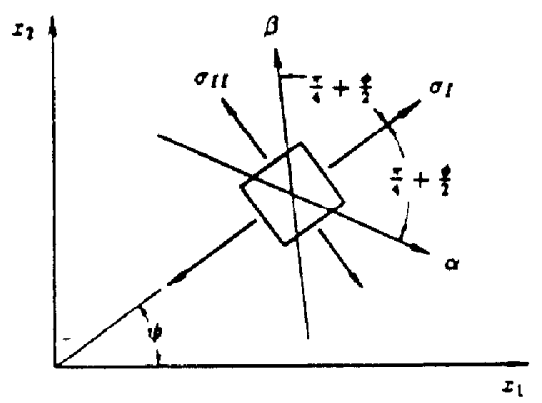

Fig. 7. Definition of characteristic lines with respect to the major and minor principal stresses $\sigma_{\text {, }}$ and $\sigma_{n}$ in the $x_{1}-x_{2}$ plane. 


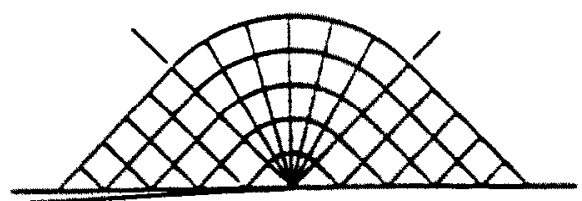

(a) $\mu=0$

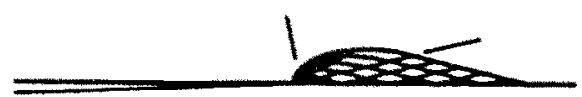

(b) $\mu=0.8$

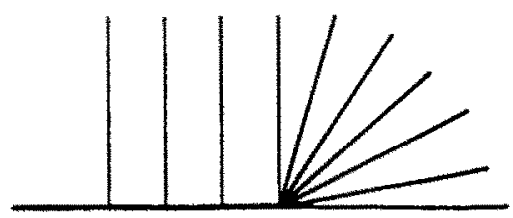

(c) $\mu-\mu_{\text {IIm }}$

Fig. 8. Crack-tip characteristic ficlus for different values of $\mu:$ (a) $\mu=0$, (b) $\mu=0.8$. (c) $\mu=\mu_{\text {hm }}=$ $\sqrt{3 / 2}$.

and for $\pi / 2<0 \leqslant \pi$ :

$$
\begin{aligned}
& \frac{\sigma_{r r}}{\sigma_{0}}=\frac{1}{3}(1+\cos 2 \theta) \\
& \frac{\sigma_{\theta \theta}}{\sigma_{0}}=\frac{1}{3}(1-\cos 2 \theta) \\
& \frac{\sigma_{\theta \theta}}{\sigma_{0}}=-\frac{1}{3} \sin 2 \theta
\end{aligned}
$$

For comparison purposes, the slip-line fields for $\mu=0$ and $\mu=0.8$ are also shown in Fig. $8 a$ and $b$, respectively. The corresponding angular distributions of the stresses normalized by $\sigma_{0}$ are shown in Fig. 9 . Note that the radial stress component $\sigma_{r r}$ becomes discontinuous along the boundary of the two sectors at $\theta=90^{\circ}$ in Fig. $9 \mathrm{c}$ for $\mu=\sqrt{3} / 2$. If we closely examine these two sectors, we find that they are actually two constant stress sectors. For $0 \leqslant \theta<\pi / 2$, the stresses are $: \sigma_{x x}=\sigma_{y y}=\sigma_{z z}=2 \sigma_{0} / 3$ and $\sigma_{x y}=0$. This sector is in hydrostatic tension. It is actually a degenerated fan sector which becomes a constant stress sector of hydrostatic tension. For $\pi / 2<\theta \leqslant \pi$, the stresses are: $\sigma_{x x}=2 \sigma_{0} / 3$ and $\sigma_{y y}=\sigma_{z:}=\sigma_{x y}=0$. This sector is in uniaxial tension in the crack line direction. The planestrain conditions is met due to the plastic dilatation in the out-of-plane direction from the normality flow based on the pressure-dependent yield condition. Due to the plane-strain constraints, the plastic strain must be zero in the front. Therefore the material must be rigid 

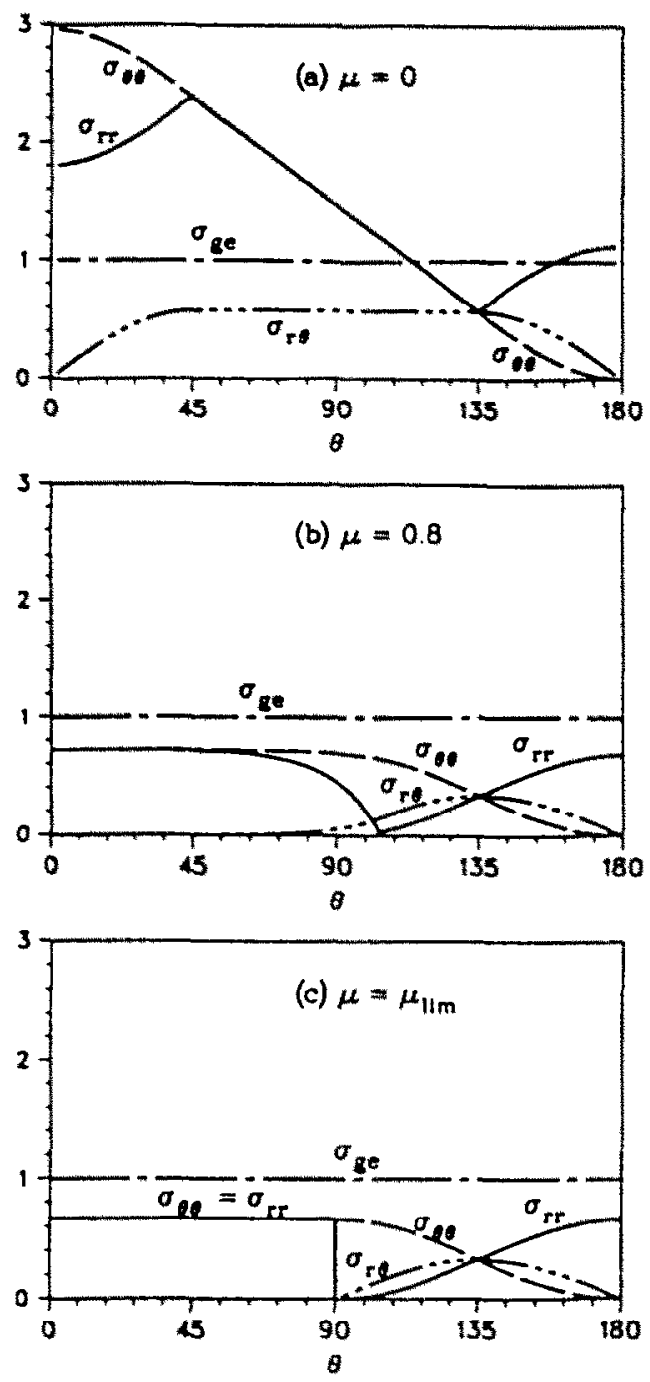

Fig. 9. Crack-tip stress fields for rigid perfectly plastic materials: (a) $\mu=0$, (b) $\mu=0.8$. (c)

$$
\mu=\mu_{1,1}=\sqrt{3}: 2
$$

in front of the crack tip. The plastic deformation occurs in uniaxial stretching in the sectors above and below the crack faces.

4.2.2. FEM results for elastic-perfectly plastic materials. Figure 10 shows the normalized stresses as functions of $\theta$ from finite element computations at $r / r_{p} \approx 10^{-2}$ for $\mu=0,0.4$ and 0.8 for elastic-perfectly plastic materials. At $r / r_{p} \approx 10^{-2}$, we obtained steady state asymptotic solutions in our computations for perfectly plastic materials. For $\mu=0$, the wellknown Prandul field is recovered (Fig. 10a). Figure $10 \mathrm{~b}$ and $\mathrm{c}$ shows the angular stress distributions for $\mu=0.4$ and 0.8 respectively. All the computational results for $\mu>0$ show that there exists an elastic sector between the upper crack surface and the neighboring partial constant stress sector. The resulting structure of the crack-tip stress field is depicted in Fig. II. Detailed discussions of the elastic-perfectly plastic crack-tip felds will be reported in Kim and Pan (1991). In contrast, for power-law hardening materials, fully yielded cracktip fields surrounding the tip are always obtained. Due to the existence of the elastic sector. the magnitudes of the opening and radial stresses ahead of the tip are slightly less than their counterparts in the rigid perfectly plastic solutions (Fig. 9).

The corresponding plastic zones normalized by $\left(1-v^{2}\right) \sigma_{0} / J E$ for $\mu=0.0 .4$ and 0.8 are shown in Fig. 12. As shown in the figure, when $\mu$ increases, the sizes and shapes of the plastic zones change drastically. As shown in Fig. $12 \mathrm{c}$ for $\mu=0.8$, the plastic zone shape 

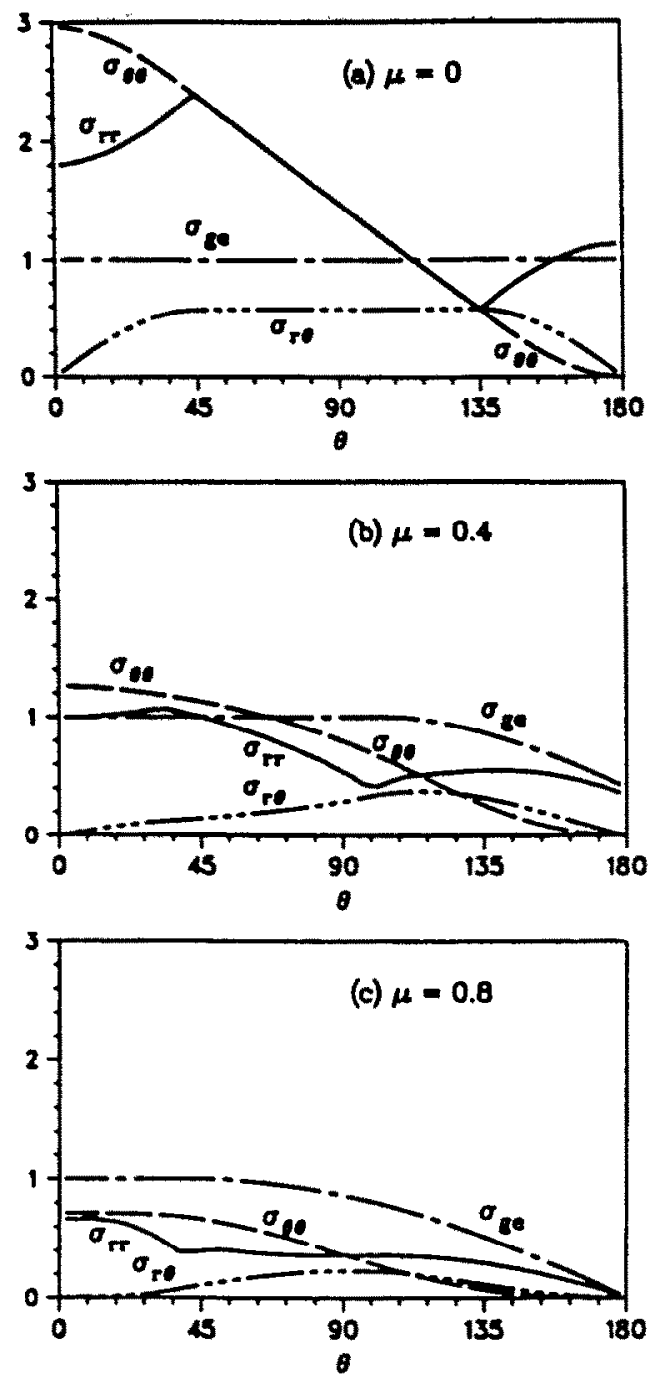

Fig. 10. Crack-tip stress fields for elastic-perfectly plastic materials from finite element computations: (a) $\mu=0$, (b) $\mu=0.4$, (c) $\mu=0.8$.

resembles the one for Mises materials under plane-stress conditions (for example, see Dong and $\mathbf{P a n}, 1990 \mathrm{~b}$ ). This indicates that pressure-sensitive yielding relieves the large hydrostatic tension ahead of the tip imposed by the plane-strain constraint conditions. As $\mu$ increases further from 0.8 , our finite element computations indicate that the angular span of the elastic sector behind the tip expands and the total angular span of the plastic sectors ahead of the tip decreases. As $\mu$ approaches $\sqrt{3} / 2$, the crack-tip field becomes elastic at all angles.

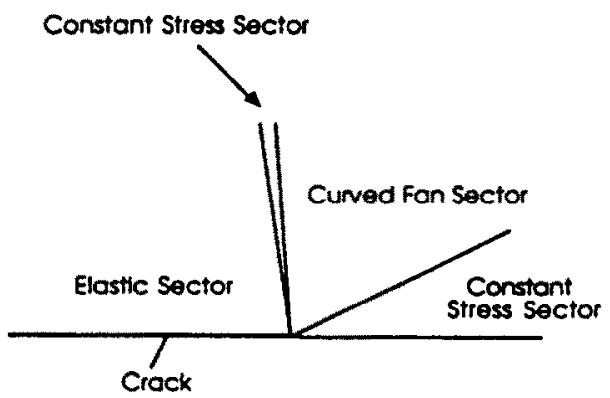

Fig. 11. Assembly of the crack-tip sectors for elastic-perfectly plastic materials with pressuresensitive yielding. 

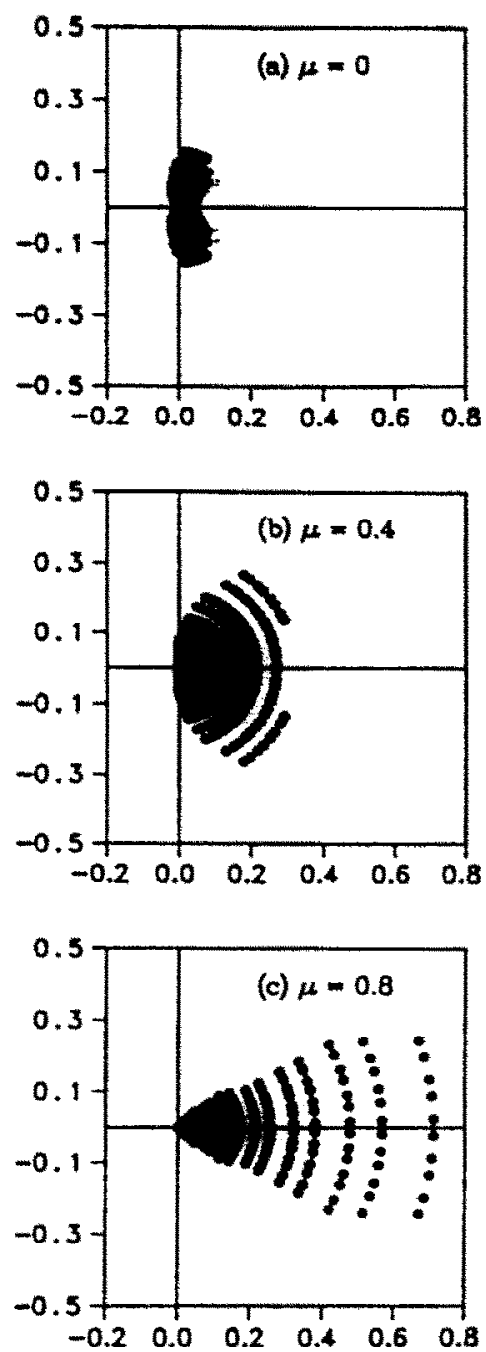

Fig. 12. Plastic zones normalized by $\left(1-v^{2}\right) \sigma_{0}^{2}, J E^{*}$ for elastic porliedly plastic materials: $(a) \mu=0$. (b) $\mu=0.4$, (c) $\mu=0.8$.

We cannot find any steady asymptotic solutions for $\mu>\sqrt{3} / 2$ for the elastic-perfectly plastic cases. It should be noted that the trend of plastic zone size and shape reported here is completely different from that of the generalized effective stress contours for large $n$ shown in Li and Pan (1990a).

\section{DISCUSSION}

Among other things, one question constantly posed throughout this investigation is whether the limit value, $\mu_{\mathrm{lm}}$, of the pressure sensitivity parameter $\mu$ indeed exists, as introduced by $\mathrm{Li}$ and Pan (1990a). Li and Pan (1990a) show that $\mu_{\mathrm{tm}}$ increases with the power-law hardening exponent. $n$, and tends to $\sqrt{3 / 2}$ as $n$ approaches $\infty$. This set of $\mu_{\text {iim }}$ apparently stems from the plane-strain constraints and the subsequent formulations in their dominant singularity analysis. As for the cases of perfectly plastic materials, the resulting equations are either hyperbolic (for $\mu<\sqrt{3} / 2$ ) or parabolic (for $\mu=\sqrt{3} / 2$ ). For the parabolic case. plastic yiclding only depends on the stress component $\sigma$ normal to the characteristic lines (Figs $6 \mathrm{e}$ and $8 \mathrm{c}$ ). Once material elasticity is introduced, the crack-tip fields for perfectly plastic materials are no longer fully plastic at all angles for $\mu>0$. The size of the elastic sector increases with the pressure sensitivity parameter $\mu$. It seems that as $\mu$ approaches $\sqrt{3} / 2$, the plastic sectors degenerate to a thin line ahead of the tip at $\theta=0$ and a completely elastic field emerges. No steady asymptotic solutions can be found for 
$\mu>\sqrt{3} 2$. This appears to be consistent with the findings discussed in Dong and Pan (1990a.b). that a discontinuity in $\sigma_{m}$, as envisioned in the rigid perfect plasticity solutions, is actually replaced by an elastic region in the same neighborhood. Since no steady solutions can be found for $\mu$ beyond $\sqrt{3} / 2$. we may conclude that the limit value of $\mu$ for the elasticperfectly plastic case is the same as the one identified for the rigid perfectly plastic case, i.e.. $\mu_{\mathrm{lm}}=\sqrt{3} 2$.

If any hardening behavior is introduced, a fully yielded HRR field is always obtained not only for $\mu<\mu_{\text {lim }}$, but also for a range of $\mu \geqslant \mu_{\text {lim }}$. This signifies that the set of $\mu_{\text {lim }}$ suggested by Li and Pan (1990a) is not the actual $\mu_{\text {lim }}$, if indeed one exists. It seems that the real limit values would be larger than those suggested by $\mathrm{Li}$ and Pan (1990a). but quantitative arguments on this issue still cannot be made without further detailed investigation.

The dramatic change in both size and shape of the plastic zones with increasing $\mu$ are shown in Figs 3, 5 and 12. As $\mu$ increases, the plastic deformation near the tip shifts to the front. This interesting feature has also been noticed in recent experiments on toughened polymer composites. where cavitation and crazing result in apparent pressure-sensitive yielding and plastic dilatation (Sue and Yee. 1989).

Acknowledgements - The computations reported were performed on an Apollo computer and in Alliant computer at the College of Engincering of The University of Michigin. The acquisition of the Apollo was made possible by a grant from the U.S. Nuclear Regulatory Commission under grant number NRC-04-87-113. J.P. acknowledges the support of this work by the National Science Foundation under grant numbers MSM-8613544 and DMR$8708+05$. Helpful discussions with A. F. Yec. I. W. Chen. and M. Kim of The University of Michigan are appricialted.

\section{REFERENCES}

Bathe. K. J. and Cimento, A. P. (1980). Some practical procedures for the solution of nonlinear finite element equations. Compur. Weth. dppl. Werh. Eingng 22, 5985.

Hen Aoun. Z. and Pant. J. ((9)1). Reseatroh to be published.

Chen, I. W. and Reyes-Morel, P. F. (1986). Implications of transformation plasticity in ZrO,-containing ceramics: I. Sheir and dilatiation eflects. J. Am. Coram. Sorc. 69, 181 189.

Dong. P. and Pan, J. (I9)(a) ). Platne-strain mixed-mode ne:ar-tip fields in elastic perfectly plastic solids under smill-sciale yiclding conditions. Int. J. Fracture 45, 243262.

Dong. P. and P'an, J. (194)(th). Planc-stress mixed-mode neir-tip tields in elastic perfectly plastic solids. Engng firculure . Mech. $37,43.57$.

Drucker, D. C. (1973). Plasticity theory, strength diflerential (SD) phenomenon, and volume expansion in metals and plastics. Mereall. Trams. $4,667673$.

Drucker, D. C. and Prager, W. (1952). Soil mechatnics and plastic analysis or limit design. Q. Appl. Math. 10, 157165 .

Gas. Y. C. (1980). Elastic plastic ficld of a crack betore growing in perfectly plastic medium [in Chinese]. Actu Solial M/ech. Sinica 1, 69-75.

llutchinsun. J. W. (1968a). Singular behaviour at the end of a tensile crack in a hardening material. J. Mech. Phy's. Solids 16, 13-31.

Hutchinson, J. W. (1968b). Plastic stress and strain fields at a crack-tip. J. Mech. Phys. Solids 16, 337-347.

Kim, M. and Pan, J. (1991). Research to be published.

Li, F. Z. and Pan, J. (1990a). Plane-strain crack-tip fields for pressure-sensitive dilatant materials. J. Appl. Mech. $57,40-49$

Li, F. Z and Pan, J. (1990b). Plane-stress crack-tip fields for pressure-sensitive dilatant materials. Engng Fracture Mech. 35, 1105-1116.

Needleman. A. and Rice, J. R. (1978). Limits to ductility set by plastic flow localization. In Mechunics of Sheet Me'tal Forming (Edited by D. P. Koistinen and N.-M. Wang), pp. 237-267. Plenum, New York.

Nematt-Nasser. S. and Obatta. M. (1984). On stress ficld near a stationary crack tip. Mech. Mater. 3, 235-243.

Reyes-Morel, P. E. and Chen, l.-W. (1988). Transformation plasticity of $\mathrm{CeO}_{2}$-stabilized tetragonal zirconia polycrystals: 1. Stress assistance and autocitalysis. J. Am. Ceram. Soc. 71(5), 343-353.

Rice. J. R. (1968). A path independent integral and the approximate analysis of strain concentration by notches and cracks. J. Appl. Mech. 35, $379 \ldots 386$.

Rice. J. R. (1982). Elastic plastic crack growth. In Mechanics of Solids: The R. Hill 60th Anniversary Volume (Fdited by H. G. Hopkins and M. J. Sewell), pp. 539-562. Pergamon Press, Oxford.

Rice. J. R. and Rosengren. G. F. (1968). Plane strain deformation near a crack tip in a power law hardening material. J. Mech. Phys. Solids 16, 1-12.

Rudnicki. J. W. and Rice. J. R. (1975). Conditions for the localization of deformation in pressure-sensitive dilatant materials. J. Mech. Phys. Solids 23, $371-394$.

Spitzig. W. A. and Richmond. O. (1979). Effect of hydrostatic pressure on the deformation behavior of polyethylene and polycarbonate in tension and in compression. Polymer Engng Sci. 19, 1129-1139.

Sue. H. J. and Yee. A. (1989). Toughening mechanisms in a multi-phase alloy of nylon 6,6/polyphenylene oxide. J. Mater. Si. 24, 1477-1457.

Wu. T. H. (1966). Sril Mechanics. Allyn \& Bacon. Boston. 